\title{
Application of fast gas chromatography and fourier transform infrared spectroscopy for analysis of lard adulteration in virgin coconut oil.
}

\begin{abstract}
Lard (LD) and virgin coconut oil (VCO) share some similarities such as having transparent to yellowish color and are solid at room temperature; hence, as a consequent, LD may be a potential oil adulterant in VCO. This study highlights the application of fast gas chromatography with surface acoustic wave detector (GC-SAW system) and Fourier transform infrared (FTIR) spectroscopy combined with chemometrics to analyze the presence of LD in VCO. Binary admixtures of LD in VCO in various percentage concentrations ranging from $1 \%$ to $50 \%(\mathrm{v} / \mathrm{v})$ were assayed using the fast GC-SAW system and FTIR spectroscopy. Using the fast GC-SAW system, ten different chromatogram peaks were identified as the adulterant peaks. One peak in the fast GC-SAW system chromatogram was found to have the best relationship, with a coefficient of determination (R 2) value of 0.9344 . Furthermore, FTIR spectroscopy coupled with partial least square (PLS) and discriminant analysis (DA) can be successfully developed for quantification and classification of LD in VCO. The results showed that PLS able to predict the LD contents in VCO with equation of $y=0.999 x+0.006$, for the correlation between actual value of LD (x) and FTIR predicted value (y) with R 2 of 0.9990 at frequency regions of 3,020-3,000 cm-1 and 1,120-1,000 $\mathrm{cm}-1$. DA can classify VCO and that adulterated with LD using the FTIR spectra at the same frequency regions used in quantification.
\end{abstract}

Keyword: Discriminant analysis; Fast GC-SAW system; FTIR spectroscopy; Lard; Partial least square; Virgin coconut oil. 